\title{
Fringe Quality Map for Fringe Projection Profilometry in LabVIEW
}

\section{Mapa de calidad de franjas para Perfilometría de Proyección de Franjas en LabVIEW}

\author{
Jesús Pineda ${ }^{1}$, Raúl Vargas ${ }^{1}$, Lenny A. Romero ${ }^{2}$, Jaime Meneses ${ }^{3}$, Andrés G. Marrugo ${ }^{1}$ \\ 1. Facultad de Ingeniería, Universidad Tecnológica de Bolívar, Cartagena, Colombia \\ 2. Facultad de Ciencias Básicas, Universidad Tecnológica de Bolívar, Cartagena, Colombia \\ 3. Grupo de Óptica y Tratamiento de Señales, Universidad Industrial de Santander, Bucaramanga, Colombia. \\ ${ }^{(*)}$ E-mail: jesuspinedacastro@outlook.com
}

Received: 10/04/2018 Accepted: 24/10/2018

DOI: 10.7149/OPA.51.4.50302

\begin{abstract}
:
The phase retrieval process is mainly affected by local shadows, irregular surface brightness and fringe discontinuities. To overcome these problems, image-processing strategies are carried out such as binary masks, interpolation techniques, and filtering. Similarly, many unwrapping algorithms have been developed to handle phase unwrapping errors in two-dimensional regions. The presence of error-prone areas can be visualized during the acquisition stage avoiding the use of image processing strategies and sophisticated phase unwrapping algorithms, which in many cases represent high computational costs and long execution times. To help overcome these problems, we propose a Fringe Quality Map based on a phase residue analysis to estimate error-prone areas during acquisition. The software was fully implemented in LabVIEW, and we provide the software as supplementary material. Experimental results demonstrate that the proposed method estimates areas with poor contrast, which lead to unwrapping errors, as well as phase errors in a more complex 3D shape.
\end{abstract}

Key words: 3D reconstruction, Fringe projection, Phase retrieval, Fringe quality map.

\section{REFERENCES AND LINKS / REFERENCIAS Y ENLACES}

[1] M. Takeda and K. Mutoh, "Fourier transform profilometry for the automatic measurement of 3-D object shapes.," Appl Opt, 22, 3977 (1983).

[2] C. Zuo, S. Feng, L. Huang, T. Tao, W. Yin, Q. Chen, "Phase shifting algorithms for fringe projection profilometry: A review," Optics and Lasers in Engineering, 109, 23-59 (2018).

[3] S. Zhang, High-Speed 3D Imaging with Digital Fringe Projection Techniques. CRC Press (2016).

[4] S. Zhang, Handbook of 3D machine vision: Optical metrology and imaging. CRC press (2013).

[5] X. Su and Q. Zhang, "Dynamic 3-D shape measurement method: a review," Optics and Lasers in Engineering, 48, 191-204 (2010).

[6] Y. Hu, Q. Chen, Y. Zhang, S. Feng, T. Tao, H. Li, W. Yin, C. Zuo, "Dynamic microscopic 3D shape measurement based on marker-embedded Fourier transform profilometry," Applied optics, 57, 772780 (2018).

[7] X. Su and W. Chen, "Reliability-guided phase unwrapping algorithm: a review," Optics and Lasers in Engineering, 42, 245-261 (2004).

[8] D. J. Bone, "Fourier fringe analysis: the two-dimensional phase unwrapping problem," Appl Opt, 30, 3627-3632 (1991).

[9] R. Vargas, J. Pineda, A. G. Marrugo, L. A. Romero, "Background intensity removal in structured light three-dimensional reconstruction," presented at the 2016 XXI Symposium on Signal Processing, Images and Artificial Vision (STSIVA), 1-6 (2016) 
[10] F. Luo, W. Chen, X. Su, "Eliminating zero spectra in Fourier transform profilometry by application of Hilbert transform," Optics Communications, 365, 76-85 (2016).

[11] D. C. Ghiglia M. D. Pritt, Two-dimensional phase unwrapping: theory, algorithms, and software, Wiley New York (1998).

[12] Budianto, D. P. K. Lun, and Y.-H. Chan, "Robust Single-shot Fringe Projection Profilometry Based on Morphological Component Analysis," IEEE Transactions on Image Processing, 27,5393-5405, (2018).

[13] J. F. Lin, X. Su, "Two-dimensional Fourier transform profilometry for the automatic measurement of three-dimensional object shapes," Optical Engineering, 34, 3297-3303 (1995).

[14] C. W. Chen, H. A. Zebker, "Phase unwrapping for large SAR interferograms: Statistical segmentation and generalized network models," IEEE Transactions on Geoscience and Remote Sensing, 40, 17091719 (2002).

[15] R. M. Goldstein, H. A. Zebker, C. L. Werner, "Satellite radar interferometry: Two-dimensional phase unwrapping," Radio science, 23, 713-720 (1988).

[16] J. Pineda, R. Vargas, L. A. Romero, J. Meneses, A. G. Marrugo, “Fringe Quality Map for Fringe Projection Profilometry in LabVIEW," figshare 2018, [retrieved 4 september 2018],

[17] A. G. Marrugo, J. Pineda, L. A. Romero, R. Vargas, J. Meneses, "Fourier Transform Profilometry in LabVIEW," Digital Systems, Asadpour Vahid (Ed.), Publisher: IntechOpen (2018).

[18] J. Barrios, M. Morón, C. Barrios, R. Contreras, A. González, and J. Meneses, "Three-dimensional scanning of the cornea by using a structured light module," Opt. Pura y Apl., 50, 351-357(2017).

\section{Introduction}

Fringe projection is a widely used technique based on structured illumination for optical three-dimensional shape measurements. There are various analysis techniques that can be employed to retrieve phase information from a pattern projected onto the surface of an object. Among the existing techniques, Fourier Transform Profilometry (FTP) [1] and Phase Shifting [2]-[4] are the most commonly used methods. FTP is extensively used when measuring dynamic objects and scenes since only a single fringe pattern is required [5], [6]. The obtained phase is wrapped in the interval $(-\pi, \pi]$, and thus a phase unwrapping algorithm is required to remove the $2 \pi$ discontinuities. This process is carried out by comparing the wrapped phase at neighboring pixels and adding or subtracting an integer number of $2 \pi$, thus obtaining the continuous phase. Phase retrieval is mainly affected by local shadows, irregular surface brightness and fringe discontinuities [7], [8]. The two boxes in Fig. 1 show areas affected by local shadows and fringe discontinuities which are common error sources in phase retrieval. To overcome these problems, several image processing strategies have been proposed such as binary masks, interpolation techniques and filtering [9], [10]. Similarly, many unwrapping algorithms have been developed to handle errors in two-dimensional phase unwrapping. A comprehensive explanation of many of these algorithms, as well as software implementation, and examples are found in Ref [11]. The presence of error-prone areas, in many cases, can be identified from the acquisition stage avoiding the use of pre-processing strategies and sophisticated phase unwrapping algorithms which in many cases represent high computational costs and long execution times [12]. To overcome these problems, we propose an acquisition strategy based on a fringe quality map to avoid sophisticated post-processing algorithms. The proposed fringe quality map is based on a calculation of phase residues to estimate potential phase unwrapping error areas. The fringe quality map is basically a $2 \mathrm{D}$ histogram in which the location of residues and quantities are indicative of the severity of the unwrapping problem and graphically revels where a phase unwrapping problem will occur.

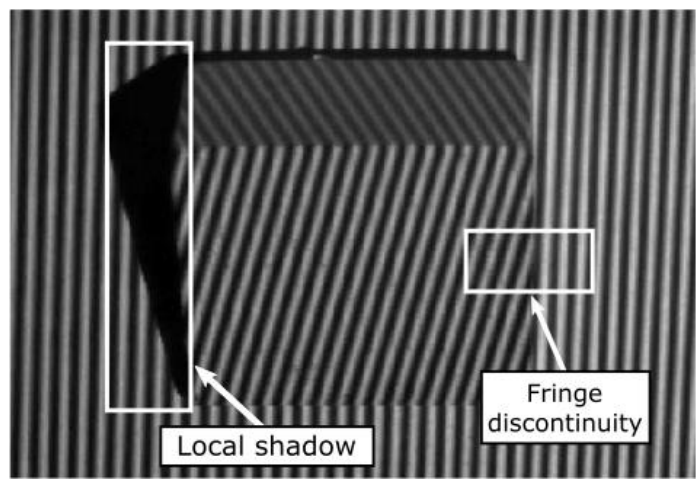




\section{Fourier Transform Profilometry}

Fig. 1. Acquired image of projected fringe pattern.

A sinusoidal grating image is projected onto the object surface. The deformed intensity fringe pattern captured by the camera can be expressed as

$$
g(x, y)=a(x, y)+b(x, y) \cos \left[2 \pi f_{0} x+\varphi(x, y)\right]
$$

where $a(x, y)$ represents the background illumination, $b(x, y)$ is the amplitude of the cosine function and relates to the contrast of the fringes, $f_{0}$ is the carrier frequency, and $\varphi(x, y)$ is the phase that contains the shape information of the object. Eq. (1) can be rewritten as

where

$$
g(x, y)=a(x, y)+c(x, y) e^{i 2 \pi f_{0} x}+c^{*}(x, y) e^{-i 2 \pi f_{0} x},
$$

$$
c(x, y)=\frac{1}{2} b(x, y) e^{i \varphi(x, y)},
$$

and superscript $*$ denotes a complex conjugate. The phase of the fringe pattern is calculated using the Fourier transform (FT) method. Using one-dimensional notation for simplicity, we can express the Fourier transform with respect to $x$ as

$$
G\left(f_{x}, y\right)=A\left(f_{x}, y\right)+C\left(f_{x}-f_{0}, y\right)+C^{*}\left(f_{x}+f_{0}, y\right)
$$

where $A\left(f_{x}, y\right)$ and $C\left(f_{x}-f_{0}, y\right)$ are the 1-D Fourier transforms of $a(x, y)$ and $c(x, y)$, respectively. $A\left(f_{x}, y\right)$ represents the zero spectra corresponding to the background component and $C\left(f_{x}-f_{0}, y\right)$ contains the shape information from the measured object. $\varphi(x, y), a(x, y)$, and $b(x, y)$ vary slowly compared to the carrier frequency $f_{0} . C^{*}\left(f_{x}+f_{0}, y\right)$ is the complex conjugate of $C\left(f_{x}-f_{0}, y\right)$.

With an appropriate filter function, for instance, a Hanning filter, the spectrum is filtered to let only the fundamental component $C\left(f_{x}-f_{0}, y\right)$. A Hanning window is described as [13]

$$
H\left(f_{x}\right)=\frac{1}{2}\left[1+\cos \left(\beta \pi \frac{f_{x}-f_{0}}{f_{c}}\right)\right]
$$

where $f_{c}$ is the cutoff frequency at a $50 \%$ attenuation ratio, $\beta=1 / 2$ and $f_{x}$ varies from $f_{0}-f_{c} / \beta$ to $f_{0}+$ $f_{c} / \beta$. The recovered signal $\hat{c}(x, y)$ by an inverse FT is given by

$$
\hat{c}(x, y)=\frac{1}{2} b(x, y) e^{i\left(\varphi(x, y)+2 \pi f_{0} x\right)} .
$$

The phase of Eq. (6) can be obtained by

$$
\Phi(x, y)=\tan ^{-1}\left(\frac{\widetilde{\Im}\{\hat{c}(x, y)\}}{\Re\{\hat{c}(x, y)\}}\right)
$$

where $\mathfrak{J}\{\hat{c}(x, y)\}$ and $\mathfrak{R}\{\hat{c}(x, y)\}$ denotes the imaginary and real part of $\hat{c}(x, y)$, respectively. The arctangent can be considered as a $2 \pi$ modulus wrapping operator. Therefore, $\Phi(x, y)$ ranges from $-\pi$ to $+\pi$. The obtained phase $\Phi(x, y)$ is called the wrapped phase map and it contains the phase of the object $\varphi(x, y)$ and the carrier phase. $\varphi(x, y)$ is often obtained by subtracting the phase of a reference plane from the recovered phase $\Phi(x, y)$. Nevertheless, the residue analysis explained in the following section can be carried out with $\Phi(x, y)$ directly.

\section{Residue Analysis}

To obtain the continuous phase, phase-unwrapping is carried out by comparing the wrapped phase at neighboring pixels and adding or subtracting an integer number of $2 \pi$. The most common assumption in unwrapping algorithms is that the wrapped neighboring-pixel phase gradients do not exceed the range of 
$(-\pi, \pi]$. If this assumption were true everywhere, for a perfect wrapped phase map the task is trivial and the unwrapped phase is an integral derivation of the original wrapped phase. Therefore, the wrapped gradients could simply be integrated along an arbitrary path to obtain the unwrapped phase map [14]. The unwrapping process is path independent if, and only if the integral around every closed path through the array is zero. The $2 \times 2$ sample path integral is the smallest closed loop that can be defined in a sampled image. In this way, the sum of unwrapped phase gradients around a closed loop of $2 \times 2$ pixels must be zero [11]. In practice, the obtained wrapped phase is not perfect and we have to deal with region for which this condition is not satisfied at all points giving rise to inconsistencies. The inconsistencies are called phase residues [15].

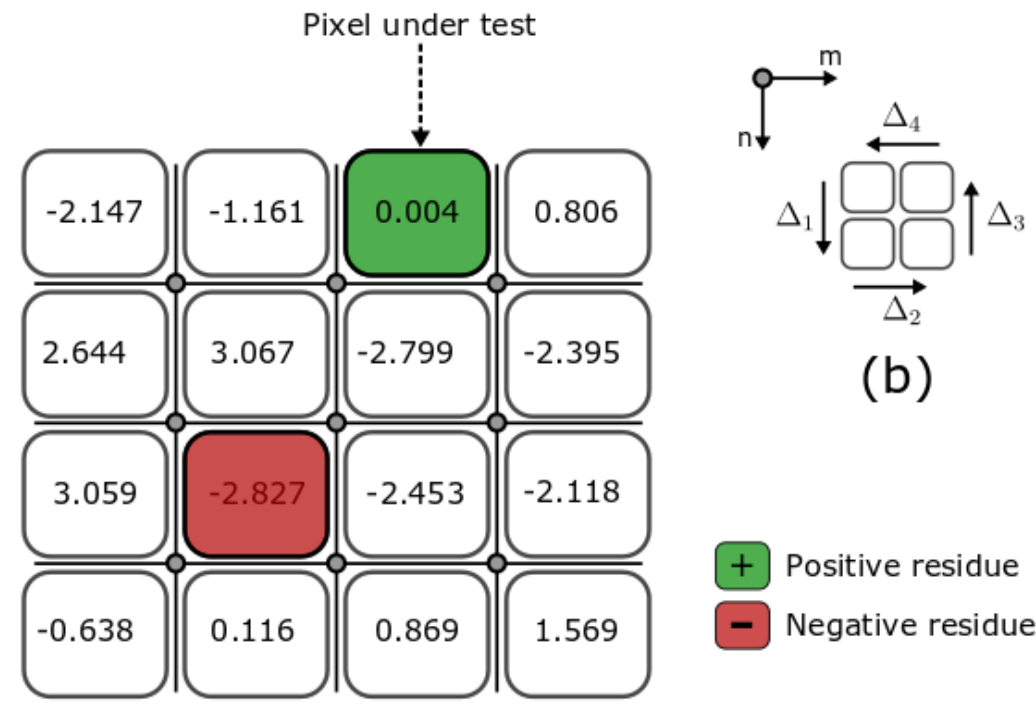

(a)

Fig. 2. Typical array of wrapped phase values.

The residue calculation process can be understood by examining the array in Fig. 2, which contains samples of a wrapped phase function $\Phi(x, y)$. The phase values in Fig. $2 \mathrm{a}$ are in units of $2 \pi$ rad and the residues are depicted as a green box for a negative residue and as a red box for a positive one. The phase derivatives are computed discretely as local wrapped two-point wrapped phase differences as shown in Fig. $2 \mathrm{~b}$. The residue charge is obtained by summing the phase differences around the closed path [8],[11] as follows,

$$
\begin{gathered}
q=\sum_{i=1}^{4} \Delta_{i}=\mathrm{W}\{\Phi(m, n+1)-\Phi(m, n)\}+\mathrm{W}\{\Phi(m+1, n+1)-\Phi(m, n+1)\}+ \\
\mathrm{W}\{\Phi(m+1, n)-\Phi(m+1, n+1)\}+\mathrm{W}\{\Phi(m, n)-\Phi(m+1, n)\}
\end{gathered}
$$

where $\mathrm{W}$ is the wrapping operator that wraps the phase differences into the interval $(-\pi, \pi]$. In the next section, we show a strategy to generate a fringe quality map based on Eq. (8) to estimate phase error-prone areas during acquisition.

\section{Proposed Method}

In this work, we propose a Fringe Quality Map (FQM) based on a phase residue analysis to identify errorprone areas during acquisition. In Fig. 3 we illustrate the proposed method. The input to the work-flow is a grabbed fringe image I. The FTP method is employed for phase retrieval. A Hanning filter is applied to perform the filtering procedure. $\mathbf{W}$ refers to the resulting wrapped phase. The zoomed region, depicted by a red box, exhibits only one of many points of phase discontinuity in $\mathbf{W}$ due to rapid surface variations of the object. We estimate the errors using Eq. (8). Notice, we are not concerned with the polarities or charges of the local integrals but in extracting $\mathbf{P}$, which refers to the location of residues around the array. Finally, we use $\mathbf{P}$ to obtain the FQM: a 2D histogram in which the location of residues and quantities are indicative of the severity of the unwrapping problem and graphically indicate where a phase unwrapping problem will occur. The 2D Histogram is a way to rapidly visualize the amount of residues in a given window. 


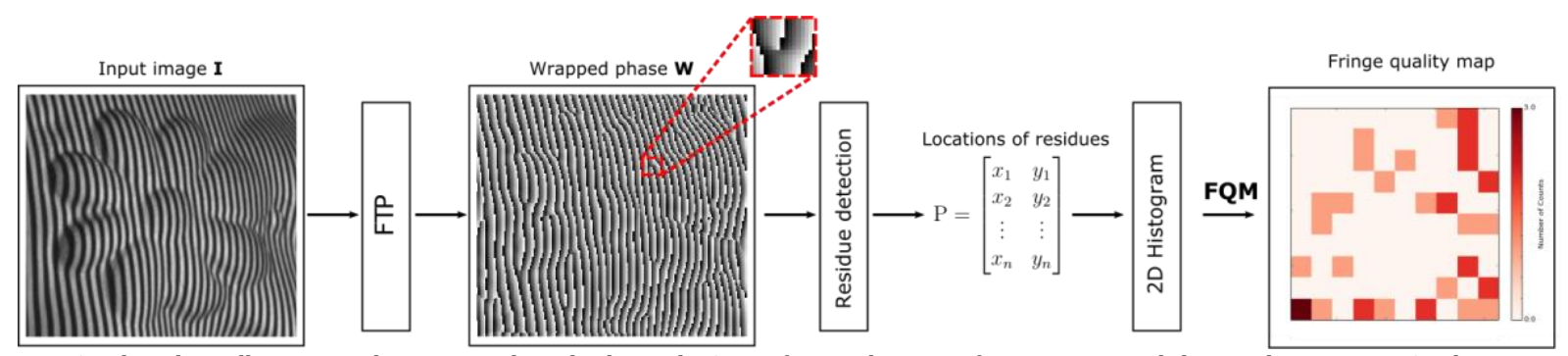

Fig. 3. Flowchart illustrating the proposed method. I and FQM refers to the input fringe image and the resulting Fringe Quality Map, respectively. The other variables are intermediate outputs of every stage; their meaning is given in the text. The software implementation of the method is available in [16].

\section{Implementation Details}

In this section, we explain the details of the software implementation in LabVIEW from National Instruments. LabVIEW is a powerful system-design platform and development environment for acquiring and analyzing data. It is highly intuitive for engineers and scientists familiar with flowcharts due to its visual programming language based on function blocks. In contrast to text-based programming languages, in graphical programming, well-written code is easier to understand and code reading is more intuitive and a whole lot quicker. LabVIEW is highly compatible with different camera hardware including USB3 Vision, GigE Vision devices, IEEE 1394 cameras compatible with IIDC, IP (Ethernet) and DirectShow compatible USB devices (e.g., cameras, webcams, microscopes, scanners). Analog cameras, digital parallel, and Camera Link, as well as NI Smart Cameras, are also supported by the software. This hardware compatibility is the main advantage of using LabVIEW for vision systems [17].

The processing strategies described in this section require the installation of the NI Vision Development Module (VDM) and the Advanced Plotting Toolkit. VDM provides image processing functions for developing machine vision applications using the LabVIEW graphical development environment. The Advanced Plotting Toolkit is an add-on package for LabVIEW to create professional-quality figures. We use this package to generate and visualize the FQM. The software implementation of the method is avialable in [16].

\section{5.a. Phase Retrieval}

The block diagram in Fig.4 illustrates the phase retrieval process in LabVIEW. Here, we use the IMAQ ImageToArray VI to extract the pixels from I into a LabVIEW 2D array for processing. FFT VI computes the fast Fourier transform (FFT) of the image array. An automatic filter design stage was implemented to filter the resulting FT spectrum. In this stage, a threshold operation is carried out to identify the location of the first order spectrum as the largest area on the right side of the binarized image. Based on the center of mass of this area, the position of the filtering window is determined. Then, we use the Inverse FFT VI to calculate the inverse discrete Fourier Transform of the filtered component. Finally, W is obtained by implementing Eq. (7). Complex To Re/Im Function breaks the complex 2-D array into its rectangular components to perform the arctangent operation using the Inverse Tangent (2 Input) Function.

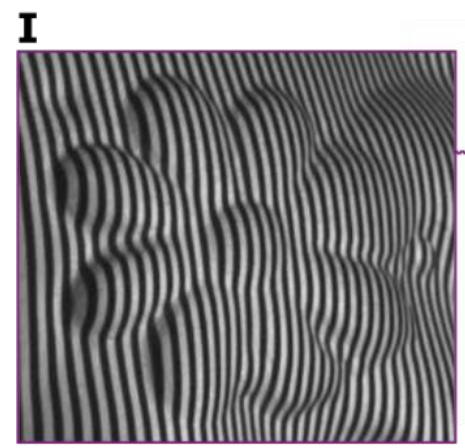

\section{FTP}

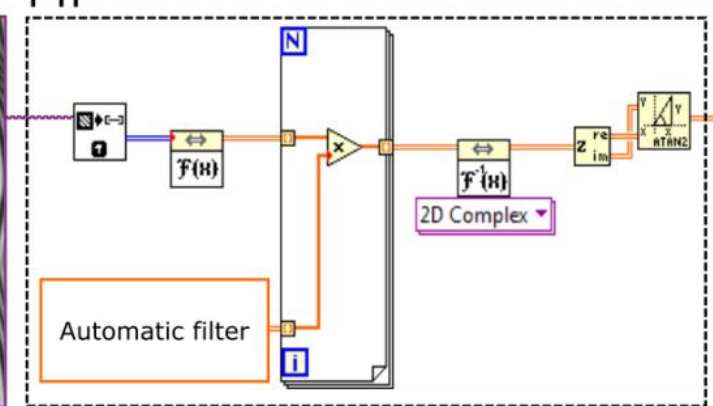

Fig. 4. Phase retrieval process in LabVIEW

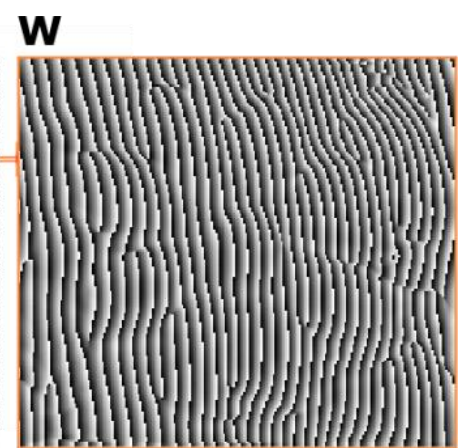

\section{5.c. Residue Detection}

In the block diagram in Fig. 5, the Residuecharge VI computes Eq. (8) throughout the entire $\mathbf{W}$-array. As previously stated, we are not interested in the residue charge $q$ but in extracting the locations of non- 
zero results. Therefore, we use the Not Equal To 0 ? Function which returns TRUE if $q$ is not equal to 0. Otherwise, it returns FALSE. Then, the Boolean FALSE or TRUE values are converts to 16-bit integers with a value of 0 or 1, respectively, using the Boolean To $(0,1)$ Function. Finally, we use the Search 1D Array Function to extract $\mathbf{P}=[\mathrm{X}, \mathrm{Y}]$, where $\mathrm{X}$ and $\mathrm{Y}$ are the horizontal and vertical locations of residues, respectively.
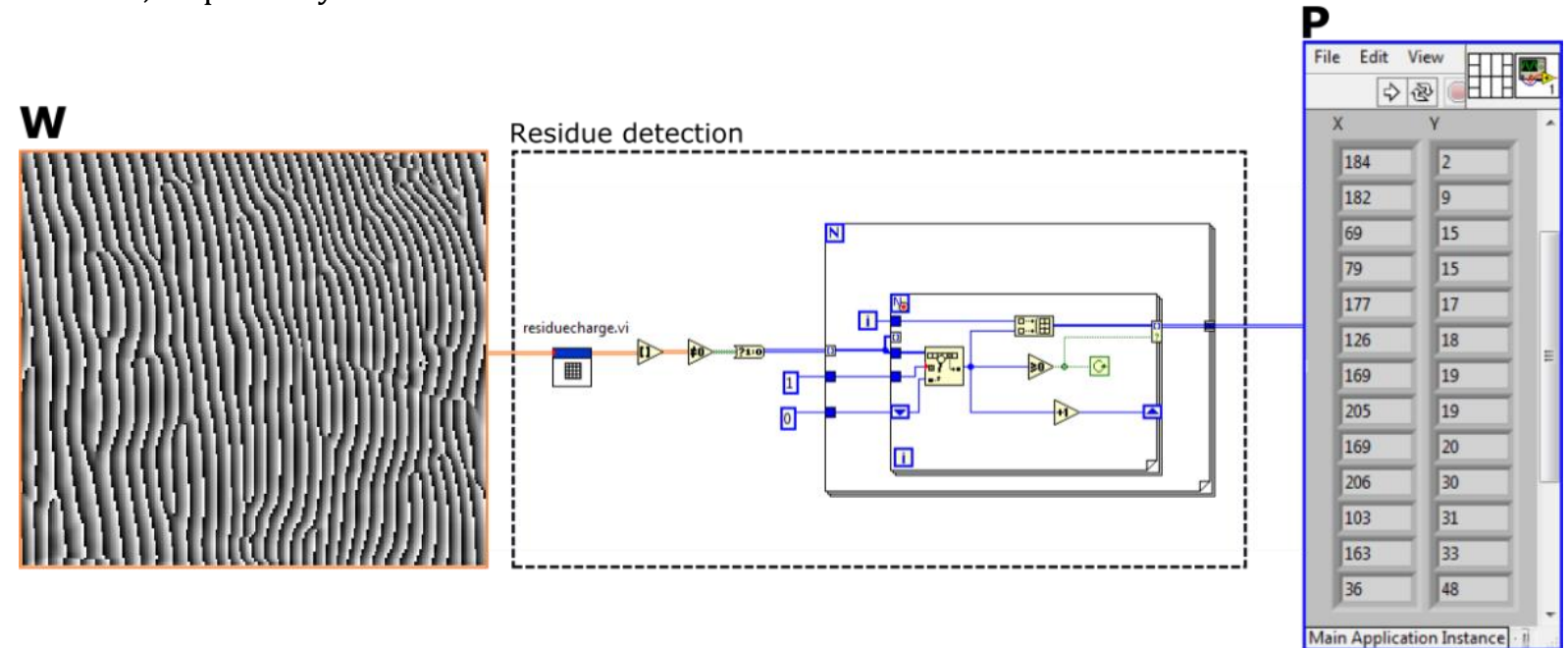

Fig. 5. Residue detection in LabVIEW.

\section{5.d. Fringe Quality Map}

We use the Advanced Plotting Toolkit to generate and visualize the FQM. In Fig. 6, the FQMplot VI relies on the 2D Histogram Plot Function to create a 2D histogram from $\mathbf{P}$. The locations of residues are supplied via the X Data and Y Data inputs using the Index Array Function. The image is divided up into 2D patches. The user must specify the number of patches desired along each axis. In the FQM, the color reflects the number of residues which fall into that patch and indicate the severity of the unwrapping problem.

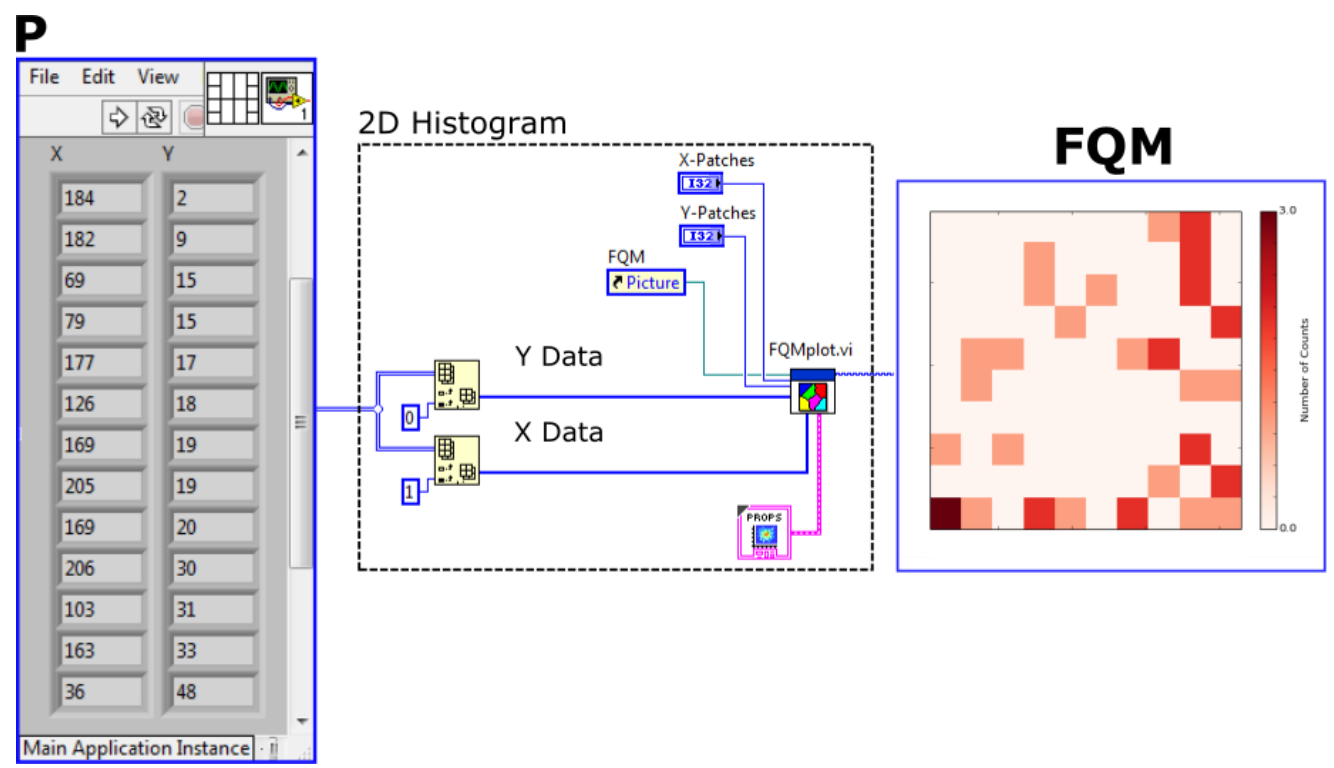

Fig. 6. Fringe quality map display in LabVIEW.

\section{Results and Discussion}

The experimental setup consisted in two parts: a projection system and an observation system. The projection system used was an LED pattern projector (Optoengineering LTPRHP3W-W)[18] that contains a stripe pattern of 400 lines with line thickness $0.01 \mathrm{~mm}$. An objective lens of $12-\mathrm{mm}$ focal length was used 
to project the fringe pattern onto the object. The observation system consists of a CMOS camera (Basler Ace with 1280x1024 pixels) that captures the distorted pattern.

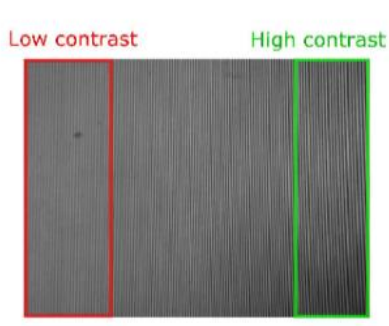

a)

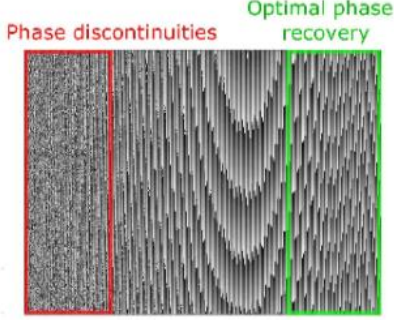

b)

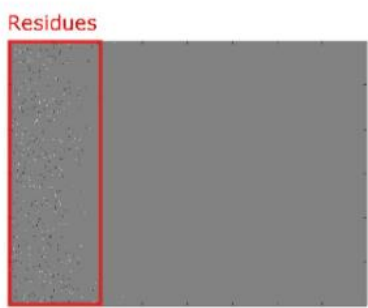

c)

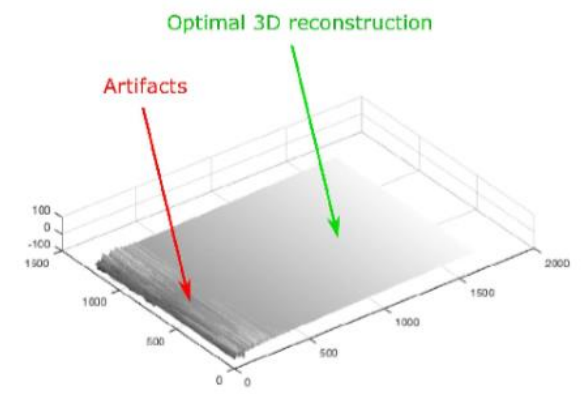

d)

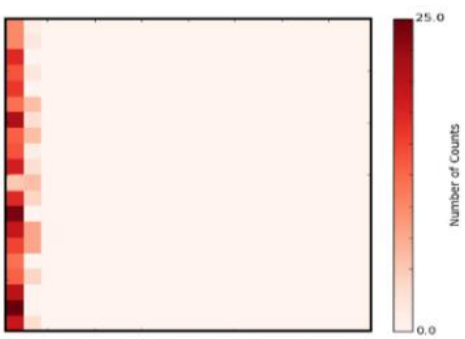

e)

Fig. 7. (a) Captured fringe pattern; (b) the wrapped phase of (a); (c) the residue map: the positive and negative residues are shown as white and black pixels, respectively; (d) 3D surface reconstruction and (e) fringe quality map.

Phase retrieval is mainly affected by local shadows, irregular surface brightness and fringe discontinuities. The fringe contrast may vary throughout the field of view of the captured image if the acquisition parameters have not been set up correctly. It is instructive to observe how this lack of contrast, together with noise and under sampling, becomes the dominant error sources in phase retrieval. Such is the case with the fringe pattern showed in Fig. 7(a), in which low and high contrast zones are surrounded by the red and green dotted lines, respectively. As shown in Fig. 7(b), the obtained wrapped phase, calculated using the Fourier transform method is affected by phase discontinuities concentrated in low contrast zones due to the poor quality and visibility of the fringe pattern that modulates the phase. The positions of residues are shown in Fig. 7(c); black indicates a negative residue and white indicates a positive residue. The Fig. 7 (d) shows the 3D reconstruction of the measured flat white board. Notice that the artifacts in the reconstructions are produced by unwrapping problems originating from the undefined phase regions in the direction of the integration path. The fringe quality map in Fig. 7(e) is basically a 2D residue density plot in which residues locations and densities are indicative of the severity of the unwrapping problem and graphically indicate where a phase unwrapping problem is likely to occur.

A more complex 3D shape can also be used to evaluate the effectiveness of the proposed method. As an example, we measured a plaster mask shown in Fig. 8(a). The fringe quality map is shown in Fig. 8(b). Once, again, residues locations visually match with the error-prone areas fairly well, as shown in the recovery 3D shape in Fig. 8(c), further confirming that residues detection algorithm works reasonably well.

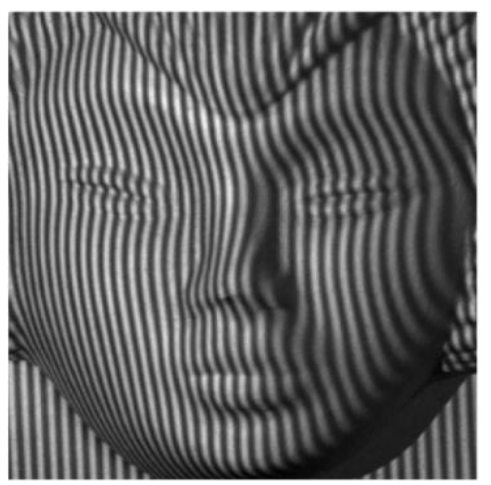

(a)

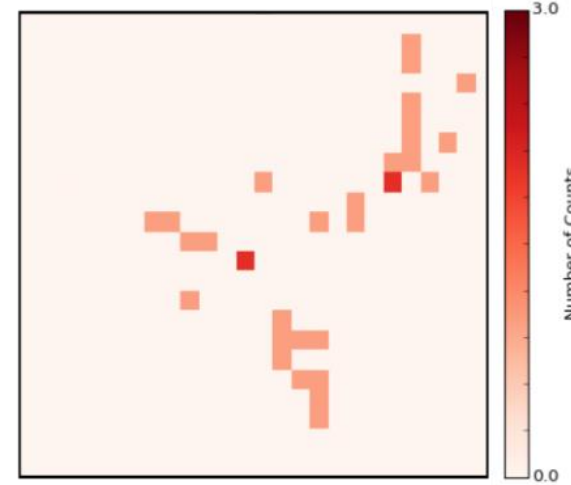

(b)

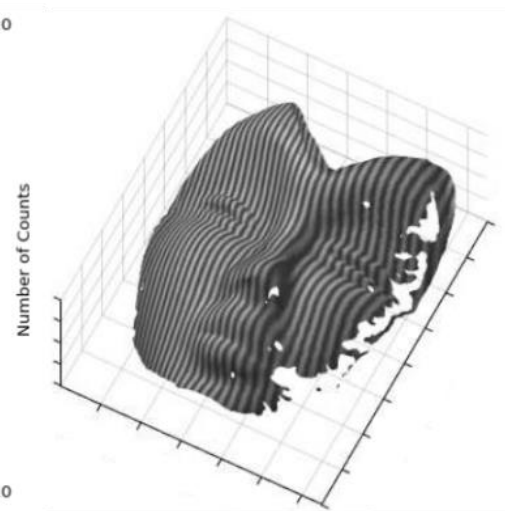

(c) 
Fig. 8. Complex 3D object measurement. (a) Captured fringe image; (b) Fringe Quality Map and (c) 3D reconstruction.

\section{Conclusions}

In this paper, we have proposed a fringe quality map method for estimating phase unwrapping problems in FTP. We have described the implementation in LabVIEW with a visualization strategy that enables to determine the severity of the unwrapping problem. We have provided the code for the FQM as supplementary material. The experimental results showed that the FQM displays areas with poor contrast, which lead to unwrapping errors, as well as phase errors in a more complex 3D shape.

\section{Acknowledgement}

This work has been partly funded by Colciencias (Fondo Nacional de Financiamiento para la Ciencia, la Tecnología y la Innovación Francisco José de Caldas) project 538871552485, and by Universidad Tecnológica de Bolívar project C2018P005. J. Pineda and R. Vargas thank Universidad Tecnológica de Bolívar for a Masters degree scholarship. Parts of this work were presented at the XV Encuentro Nacional de Óptica y VI Conferencia Andina y del Caribe en Óptica y sus aplicaciones (20-24 November; Bucaramanga, Colombia, 2017). 